\title{
The Regulation of Surface-Enhanced Raman Scattering Sensitivity of Silver Nanorods by Silicon Sections
}

\author{
Xian Zhang, ${ }^{1}$ Qin Zhou, ${ }^{1,2}$ Yu Huang, ${ }^{1}$ Zhengcao Li, ${ }^{1}$ and Zhengjun Zhang ${ }^{1}$ \\ ${ }^{1}$ Key Laboratory of Advanced Materials (MOE), School of Materials Science and Engineering, \\ Tsinghua University, Beijing 100084, China \\ ${ }^{2}$ Institute of Nuclear and New Energy Technology, Tsinghua University, Beijing 100084, China
}

Correspondence should be addressed to Zhengjun Zhang; zjzhang@tsinghua.edu.cn

Received 26 April 2013; Revised 6 July 2013; Accepted 10 July 2013

Academic Editor: Miguel A. Correa-Duarte

Copyright (c) 2013 Xian Zhang et al. This is an open access article distributed under the Creative Commons Attribution License, which permits unrestricted use, distribution, and reproduction in any medium, provided the original work is properly cited.

\begin{abstract}
Vertically aligned silver nanorods were good substrates for surface-enhanced Raman scattering. The surface-enhanced Raman scattering sensitivity of nanorods can be regulated through the method that the silver nanorod is divided into four uniform silver sections using five uniform silicon sections. And the length of silicon sections is the key factor in regulating the surface-enhanced Raman scattering sensitivity. In the regulation, the best surface-enhanced Raman scattering performance is about 4 times as large as the worst performance. The study provides an effective way to regulate the surface-enhanced Raman scattering sensitivity of silver nanorods and its possible explanation about mechanism.
\end{abstract}

\section{Introduction}

Surface-enhanced Raman scattering (SERS) is one of the most promising tools in detection at trace level of pollutants and biochemicals, due to its ultrasensitivity, convenience, and cheapness [1-4]. SERS was first discovered in 1974 that the intensity of Raman spectrum from organic molecules can be enhanced when molecules are adsorbed on the surface of silver electrode [2-5]. This effect has been verified in many kinds of metals such as silver, gold, copper, lithium, sodium, and potassium $[1-4,6]$. The magnification of Raman intensity can be mainly attributed to electromagnetic mechanism and chemical mechanism $[7,8]$. SERS seems to be obvious on surface that contains many coupled metal domains, referred to as SERS-active surface [9]; thus, devising ways has been a focus in the field to improve the performance of SERS substrates.

A quantity of structures have been discovered with good SERS sensitivity, such as the electrode surface formed by metals' solution and redeposition into clusters [9] periodically arranged gold particles with hierarchical rough surface by electrochemical deposition [10] metal colloid, and so forth [6, 11-13]. A powerful means worthwhile mentioning in producing SERS substrate is the glancing angle deposition (GLAD) technique, which is capable of producing one-dimensional
(1D) nanostructures of various morphologies [14-17]. For instance, slanted or vertically aligned silver nanorods on planar substrates can be deposited by this technique, which are proved to be excellent SERS substrates to detect organic molecules [3, 14-18]. A typical SEM image is shown from Figure 1(a) of the morphology of Ag nanorods deposited on planar silicon substrates. It is found that the silver nanorods are well vertically aligned and separated, with diameter of several ten nanometers. It is shown from Figure 1(b) that Raman spectrum of R6G molecules at trace level on this substrate can be detected $[14,15,17]$.

The sensitivity of SERS substrate is one of the most important factor in the field of SERS, while much attention has been paid to develop effective methods to regulate the sensitivity of SERS substrate. It is reported that nanostructures arranged periodically might result in different localized electric field from that of nanostructures arranged randomly $[2,19,20]$. While vertically silver nanorods from GLAD technique are proved to be SERS substrate with excellent sensitivity, it is of great interest to reform each silver nanorod into periodical structure and investigate the effect of nanorods' reformation on SERS sensitivity. In the study, five uniform silicon sections with specific length are brought in to separate the silver nanorod into four uniform silver sections. And it is found that 


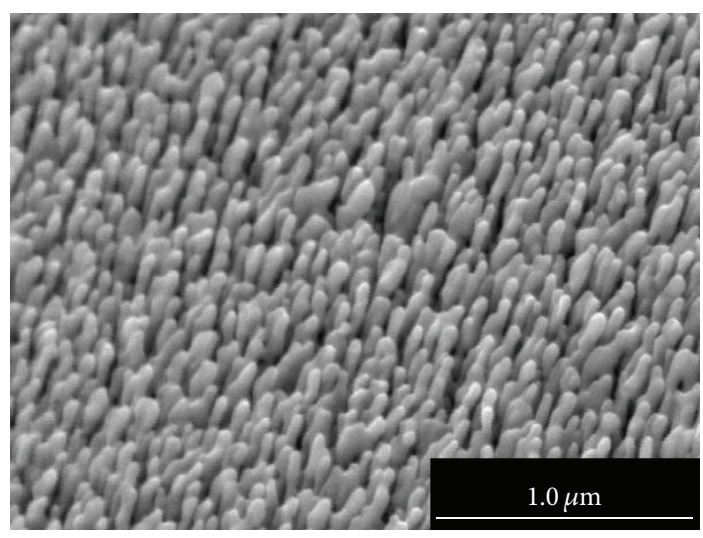

(a)

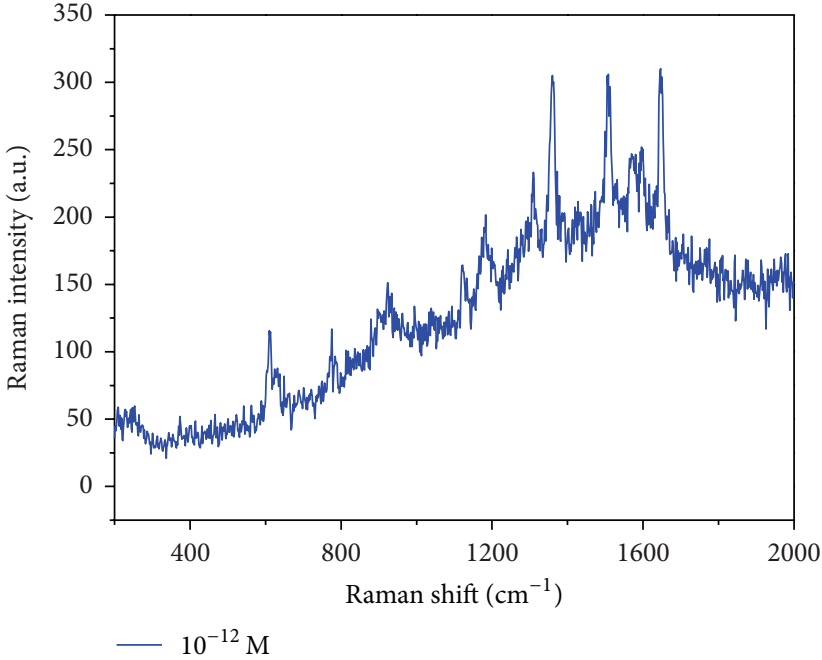

(b)

Figure 1: (a) The SEM image of vertically aligned Ag nanorods deposited on planar silicon substrates and (b) Raman spectra of R6G on these Ag nanorods as the SERS substrate, at a concentration of $10^{-12} \mathrm{M}$.

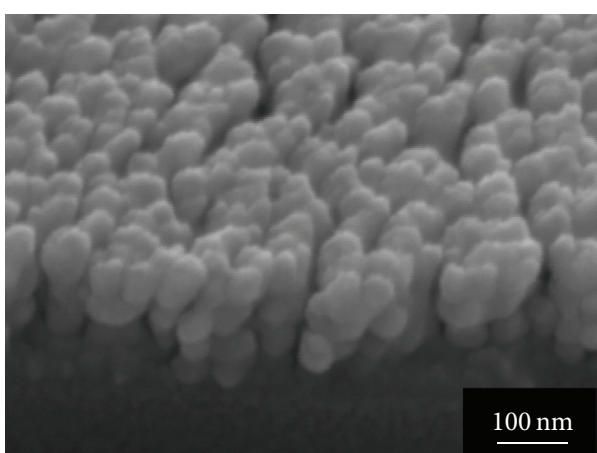

(a)

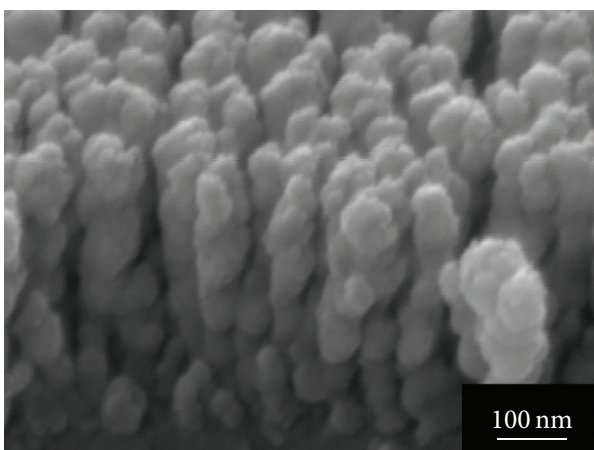

(c)

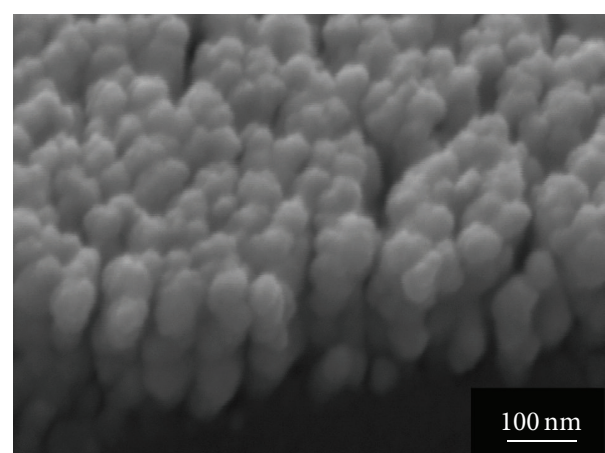

(b)

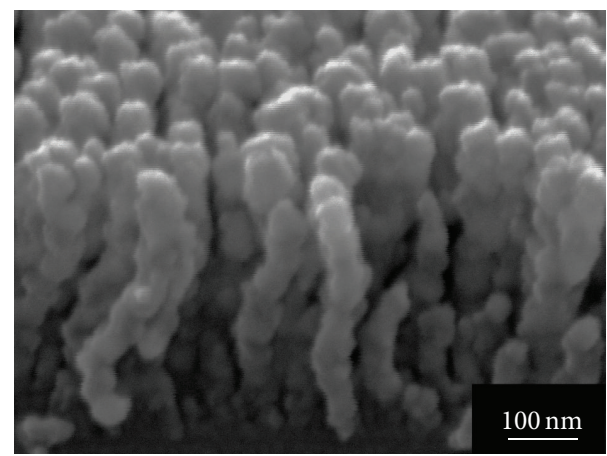

(d)

FIGURE 2: Oblique-view SEM images of vertically aligned nanorods consisted of four silver sections with length of $25 \mathrm{~nm}$ and silicon sections with length of (a) $10 \mathrm{~nm}$, (b) $20 \mathrm{~nm}$, (c) $40 \mathrm{~nm}$, and (d) $60 \mathrm{~nm}$, respectively.

the SERS sensitivity can be regulated by the length of silicon sections when the length of the silver section is fixed.

\section{Materials and Methods}

The substrates which nanorods grow on in this study were pristine (100) silicon substrates. The silicon substrate was cleaned in sequence, supersonically in acetone, ethanol, and deionized water baths and was then mounted on the substrate holder in e-beam deposition system (GLAD SYSTEM, Thermionics Laboratory, Inc., with a background vacuum level better than $2 \times 10^{-5} \mathrm{~Pa}$ ). Vertically aligned nanorods were then deposited on the substrate by the GLAD technique. During deposition, the substrate was cooled to $-20^{\circ} \mathrm{C}$ by 


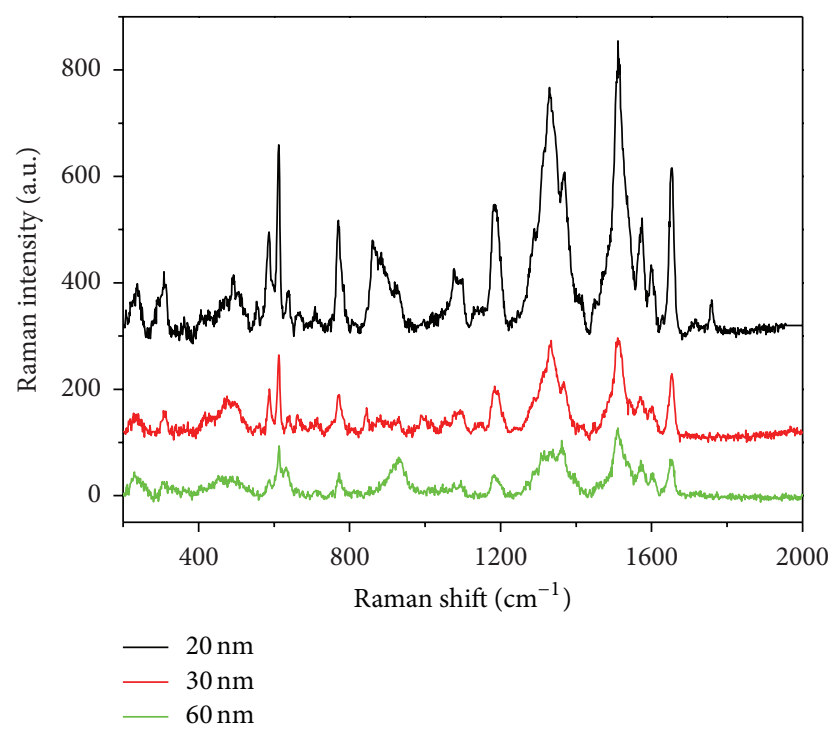

FIGURE 3: Raman spectra of R6G (concentration of $10^{-6} \mathrm{M}$ ), characterized by aligned nanorods, consisted of four silver sections with $25 \mathrm{~nm}$ length and five silicon sections with length of $20 \mathrm{~nm}$, $30 \mathrm{~nm}$ and $60 \mathrm{~nm}$, respectively; the Raman spectra are substracted by baseline and added by constant line to make the three Raman spectra separated.

liquid nitrogen, revolving on its axis at a speed of $2 \mathrm{rpm}$. The angle was set $\sim 88^{\circ}$ between surface normal of substrate and the incoming vapor flux, while the deposition rate was $\sim 0.5 \mathrm{~nm} / \mathrm{s}$ monitored by a quartz crystal microbalance.

The film of nanorods is deposited in turn with silver sections and silicon sections. For one nanorod, first of all, one silicon section is deposited on the substrate with a specific length, then the silver section with length of $25 \mathrm{~nm}$ is deposited on the silicon section, and so on. In the end, nanorod is produced with five uniform silicon sections with specific length and four silver sections with length of $25 \mathrm{~nm}$, so the final silicon section is the top of the whole nanorod. The nanorod can be considered as four silver sections separated by five silicon sections; meanwhile, the top and bottom of the nanorod are both silicon sections. Moreover, the length of uniform silicon sections in nanorods is designed to be $10 \mathrm{~nm}$, $20 \mathrm{~nm}, 30 \mathrm{~nm}, 40 \mathrm{~nm}, 50 \mathrm{~nm}$, and $60 \mathrm{~nm}$.

The morphology of nanorods with silver sections and silicon sections was characterized by scanning electron microscopy (JEOL JSM-7001F). The performance of nanorods as SERS substrate was evaluated by a Renishaw 100 Raman spectrometer using Rhodamine 6G (R6G) as the probing molecule. Before the Raman scattering measurements, all SERS substrates were dipped into aqueous solutions of $1 \times$ $10^{-6} \mathrm{M}$ R6G for 30 minutes and dried by a gentle continuous nitrogen blow. Then, the experimental Raman spectra of R6G on each substrate were obtained by Raman spectrometer using $633 \mathrm{~nm} \mathrm{He-Ne} \mathrm{laser} \mathrm{as} \mathrm{the} \mathrm{excitation} \mathrm{source.} \mathrm{During}$ measurements, the laser power was decreased to $0.47 \mathrm{~mW}$ to avoid any damage to R6G molecules, with signal accumulation time of 15 second per $600 \mathrm{~cm}^{-1}$ and 50x objective lens. However, the Raman spectrum for analysis and process of R6G on specific substrate is derived by averaging experimental Raman spectra from six different areas on the same SERS substrate, to minimize the error from measurement. The SERS performance of nanorods is generated from silver sections because silicon can generate no SERS effect.

\section{Results and Discussion}

Tilt-view SEM images are shown in Figure 2, of nanorods consisted of four silver sections with length of $25 \mathrm{~nm}$ and silicon sections with lengths of $10 \mathrm{~nm}, 20 \mathrm{~nm}, 40 \mathrm{~nm}$, and $60 \mathrm{~nm}$ respectively. It is found that each nanorod with about $70 \mathrm{~nm}$ diameter is divided into sections, and the length of nanorod in different samples is variable owing to different length of silicon sections. The pixel length ratio of nanorods in the four samples is about $1: 1.31: 2.02: 2.45$, approximately the same as $1: 1.33: 2.00: 2.66(150: 200: 300: 400)$, which is the designed length ratio of nanorods in the four samples.

Raman spectra are shown in Figure 3 of R6G molecules with concentration of $1 \times 10^{-6} \mathrm{M}$, which are measured from nanorods with silicon sections' length of $20 \mathrm{~nm}, 30 \mathrm{~nm}$, and $60 \mathrm{~nm}$. It is shown that each spectrum presents the Raman features of R6G molecules and that the signal intensity of the Raman spectrum is very dependent on the length of silicon sections in nanorods. For example, the signal intensity is the highest, when the length of silicon sections is $20 \mathrm{~nm}$, and is the lowest, when the length of silicon sections is $60 \mathrm{~nm}$. Difference in the Raman intensity is noticed among films of nanorods consisted of silicon sections with different length. Although the amount of silver section is fixed, the SERS sensitivity of nanorods can be regulated by the length of silicon sections. And the reproducibility of Raman spectra is shown in Figures 4(a) and 4(b), respectively, by the average value and standard deviation of experimental Raman spectra (after baseline correction) from six different areas on nanorods consisted of silicon sections with $50 \mathrm{~nm}$ length.

To gain a better understanding of the law how the length of silicon sections regulates the SERS sensitivity of nanorods, the intensity of one characteristic peaks of R6G molecules, peak $612 \mathrm{~cm}^{-1}$ which is assigned to the xanthene ring stretching vibration mode [21,22], is plotted as a function of the length of silicon sections. And the intensity of $612 \mathrm{~cm}^{-1}$ peak versus the length of silicon sections in nanorods is shown in Figure 5(a). It is inferred roughly that the SERS sensitivity of film is getting weaker when the length of silver sections is fixed and the length of silicon sections is getting larger. By careful observation, it is found that the intensity of peak $612 \mathrm{~cm}^{-1}$ is highest when the length of silicon sections is $20 \mathrm{~nm}$, while the intensity of peak $612 \mathrm{~cm}^{-1}$ is weakest when the length of silicon sections is $60 \mathrm{~nm}$. The intensity of peak $612 \mathrm{~cm}^{-1}$ from the film with silicon sections of $20 \mathrm{~nm}$ length is 3.8 times as large as that from the film with silicon sections of $60 \mathrm{~nm}$ length. It is proved that changing the length of silicon sections is an effective way to regulate the SERS sensitivity of film of nanorods.

The amount and morphology of four silver sections is unique in different films, while SERS cannot be generated from silicon sections. It is interesting to investigate the reason 


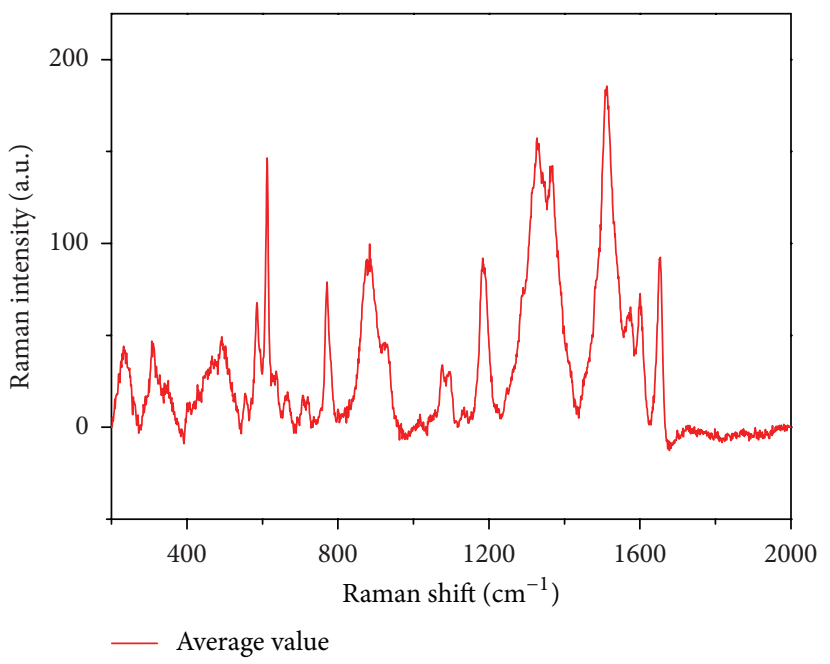

(a)

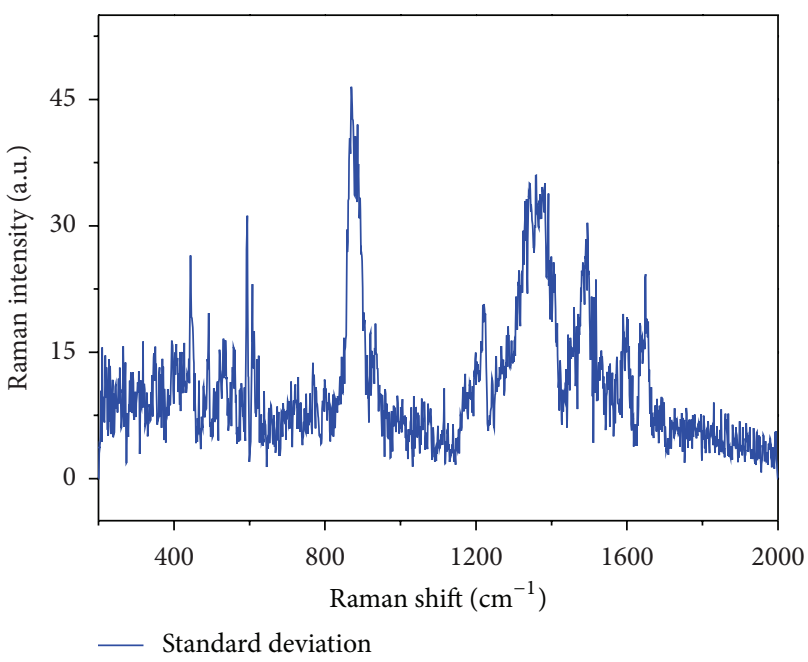

(b)

Figure 4: (a) Average value and (b) standard deviation, of experimental Raman spectra (after baseline correction) of R6G measured from six different areas on nanorods consisted of silicon sections with $50 \mathrm{~nm}$ length.

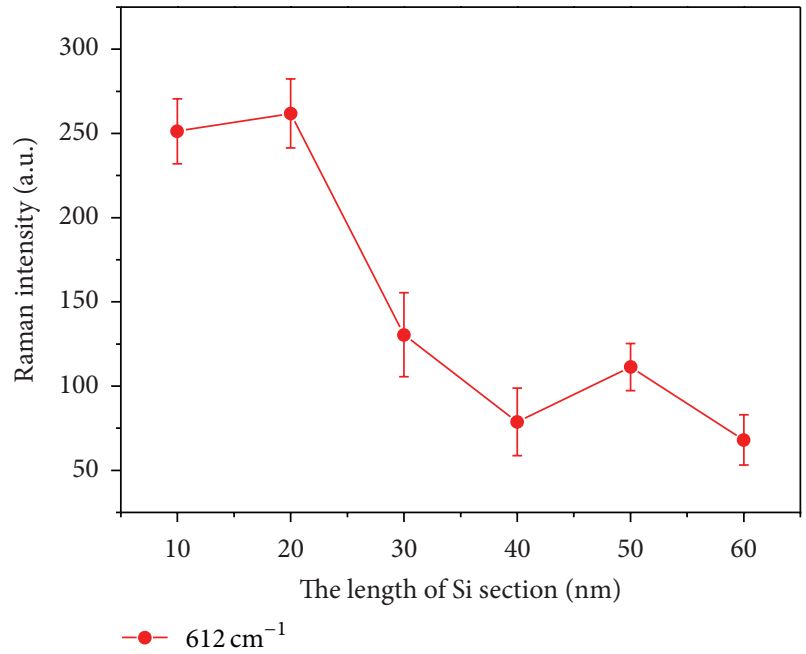

(a)

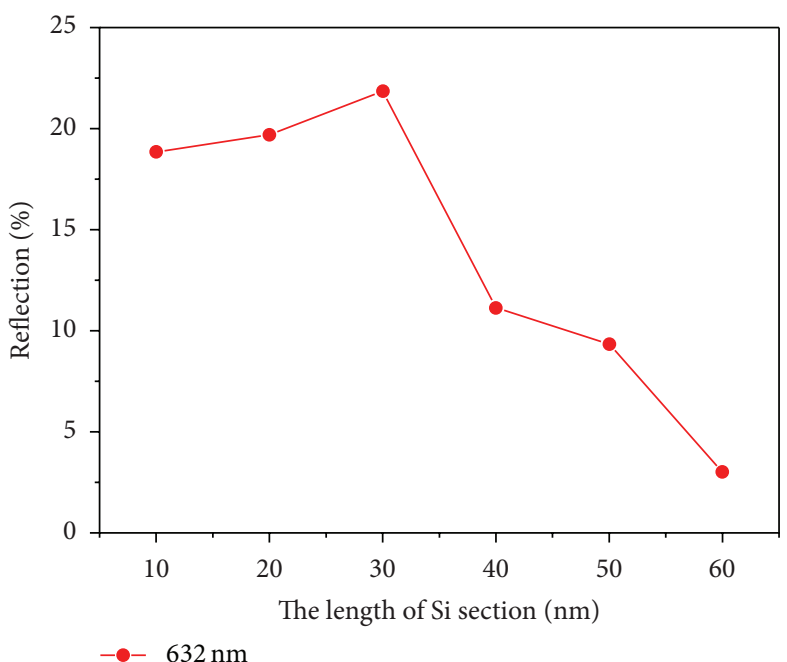

(b)

FIGURE 5: (a) The intensity of the characteristic peak $612 \mathrm{~cm}^{-1}$ from R6G on films of nanorods consisted of silver sections and silicon sections and (b) the reflection of the films versus the length of silicon sections in nanorods, when the wavelength of incident light is $632 \mathrm{~nm}$.

why the different films of nanorods have different SERS sensitivity which have fixed silver sections and different silicon sections. It is shown in Figure 5(b) that the reflection of films of nanorods is getting larger at first and getting smaller later as the length of silicon sections is getting larger, when the wavelength of incident light is $632 \mathrm{~nm}$ approximately the same as the wavelength of incident laser in Raman measurement. So as the length of silicon sections is getting larger, the absorption of nanorods is getting larger overall, but the SERS sensitivity is getting weaker overall. So it is probable that the length of silicon sections has effects on the absorption of silicon sections and even the amount of incident laser photon that silver sections can accept. The regulation of SERS sensitivity of nanorods may be probably caused by the difference of absorption of silicon sections. Moreover, it is reported that SERS performance of nanostructure consisted of periodical blocks can be tuned $[4,6,23-26]$. While the separation distance of periodical uniform silver sections is the length of silicon sections, the interaction between the localized surface plasmon electrical fields of silver sections may be a probable factor that regulate, the SERS sensitivity of nanorods.

\section{Conclusion}

In this paper, it is reported that, by dividing the silver nanorod into four silver sections with $25 \mathrm{~nm}$ length using five uniform silicon sections with specific length, the SERS sensitivity of 
nanorods can be regulated and that the SERS sensitivity of nanorods is getting weaker overall when the length of silicon sections is getting larger. The regulation of SERS sensitivity is realized possibly by the absorption of silicon sections or interaction between localized surface plasmon electrical fields of silver sections.

\section{Acknowledgments}

This work was financially supported by the National Basic Research Program of China (973 program, Grant no. 2013CB934301), by the National Natural Science Foundation of China (Grant nos. 50931002 and 51228101), and by Tsinghua University Initiative Scientific Research Program.

\section{References}

[1] Z. L. Huang, G. W. Meng, Q. Huang, Y. J. Yang, C. H. Zhu, and C. L. Tang, "Improved SERS performance from Au nanopillar arrays by abridging the pillar tip spacing by Ag sputtering," Advanced Materials, vol. 22, no. 37, pp. 4136-4139, 2010.

[2] R. J. C. Brown and M. J. T. Milton, "Nanostructures and nanostructured substrates for surface-enhanced Raman scattering (SERS)," Journal of Raman Spectroscopy, vol. 39, no. 10, pp. 13131326, 2008.

[3] R. A. Tripp, R. A. Dluhy, and Y. Zhao, "Novel nanostructures for SERS biosensing," Nano Today, vol. 3, no. 3-4, pp. 31-37, 2008.

[4] M. Moskovits, "Surface-enhanced spectroscopy," Reviews of Modern Physics, vol. 57, no. 3, pp. 783-826, 1985.

[5] M. Fleischmann, P. J. Hendra, and A. J. McQuillan, "Raman spectra of pyridine adsorbed at a silver electrode," Chemical Physics Letters, vol. 26, no. 2, pp. 163-166, 1974.

[6] A. Kudelski, "Analytical applications of Raman spectroscopy," Talanta, vol. 76, no. 1, pp. 1-8, 2008.

[7] S. F. Fischer and R. P. Van Duyne, "On the theory of electron transfer reactions. The naphthalene-/TCNQ system," Chemical Physics, vol. 26, no. 1, pp. 9-16, 1977.

[8] M. G. Albrecht and J. A. Creighton, "Anomalously intense Raman spectra of pyridine at a silver electrode," Journal of the American Chemical Society, vol. 99, no. 15, pp. 5215-5217, 1977.

[9] K. Hering, D. Cialla, K. Ackermann et al., "SERS: a versatile tool in chemical and biochemical diagnostics," Analytical and Bioanalytical Chemistry, vol. 390, no. 1, pp. 113-124, 2008.

[10] G. T. Duan, W. P. Cai, Y. Y. Luo, Y. Li, and Y. Lei, "Hierarchical surface rough ordered Au particle arrays and their surface enhanced Raman scattering," Applied Physics Letters, vol. 89, no. 18, Article ID 181918, 2006.

[11] K. Kneipp, Y. Wang, H. Kneipp et al., "Single molecule detection using surface-enhanced Raman scattering (SERS)," Physical Review Letters, vol. 78, no. 9, pp. 1667-1670, 1997.

[12] X.-M. Qian and S. M. Nie, "Single-molecule and single-nanoparticle SERS: from fundamental mechanisms to biomedical applications," Chemical Society Reviews, vol. 37, no. 5, pp. 912920, 2008.

[13] S. M. Nie and S. R. Emory, "Probing single molecules and single nanoparticles by surface-enhanced Raman scattering," Science, vol. 275, no. 5303, pp. 1102-1106, 1997.

[14] Q. Zhou, Z. C. Li, Y. Yang, and Z. J. Zhang, "Arrays of aligned, single crystalline silver nanorods for trace amount detection," Journal of Physics D, vol. 41, no. 15, Article ID 152007, 2008.
[15] Q. Zhou, Y. Yang, J. Ni, Z. Li, and Z. Zhang, "Rapid detection of 2, 3, 3', 4, 4' -pentachlorinated biphenyls by silver nanorodsenhanced Raman spectroscopy," Physica E, vol. 42, no. 5, pp. $1717-1720,2010$

[16] Y.-J. Liu, H. Y. Chu, and Y.-P. Zhao, "Silver nanorod array substrates fabricated by oblique angle deposition: morphological, optical, and SERS characterizations," Journal of Physical Chemistry C, vol. 114, no. 18, pp. 8176-8183, 2010.

[17] Y.-J. Liu, Z.-Y. Zhang, R. A. Dluhy, and Y.-P. Zhao, “The SERS response of semiordered Ag nanorod arrays fabricated by template oblique angle deposition," Journal of Raman Spectroscopy, vol. 41, no. 10, pp. 1112-1118, 2010.

[18] Q. Zhou, Y. Yang, J. Ni, Z. Li, and Z. Zhang, "Rapid recognition of isomers of monochlorobiphenyls at trace levels by surfaceenhanced Raman scattering using Ag nanorods as a substrate," Nano Research, vol. 3, no. 6, pp. 423-428, 2010.

[19] C. L. Haynes, A. D. McFarland, and R. P. Van Duyne, "Surfaceenhanced: Raman spectroscopy," Analytical Chemistry, vol. 77, no. 17, pp. 338A-346A, 2005.

[20] R. A. Alvarez-Puebla, A. Agarwal, P. Manna et al., "Gold nanorods 3D-supercrystals as surface enhanced Raman scattering spectroscopy substrates for the rapid detection of scrambled prions," Proceedings of the National Academy of Sciences of the United States of America, vol. 108, no. 20, pp. 8157-8161, 2011.

[21] P. Hildebrandt and M. Stockhurger, "Surface-Enhanced Resonance Raman Spectroscopy of Rhodamine 6G adsorbed on colloidal silver," Journal of Physical Chemistry, vol. 88, no. 24, pp. 5935-5944, 1984.

[22] H. Watanabe, N. Hayazawa, Y. Inouye, and S. Kawata, "DFT vibrational calculations of Rhodamine $6 \mathrm{G}$ adsorbed on silver: analysis of tip-enhanced Raman spectroscopy," Journal of Physical Chemistry B, vol. 109, no. 11, pp. 5012-5020, 2005.

[23] S. Acquah, D. Ventura, and H. Kroto, "On paper the future is rosy," Chemistry and Industry, no. 22, pp. 22-24, 2011.

[24] X. Zhang, Q. Zhou, J. Ni, Z. Li, and Z. Zhang, "Surface-enhanced Raman scattering from a hexagonal lattice of micro-patterns of vertically aligned Ag nanorods," Physica E, vol. 44, no. 2, pp. 460-463, 2011

[25] Y. Q. Zhu, R. L. D. Whitby, R. Ma, and S. F. A. Acquah, "1D nanomaterials 2011," Journal of Nanomaterials, vol. 2012, Article ID 535697, p. 2, 2012.

[26] X. Zhang, Q. Zhou, W. Wang, L. Shen, Z. Li, and Z. Zhang, "Latticing vertically aligned Ag nanorods to enhance its SERS sensitivity," Materials Research Bulletin, vol. 47, no. 3, pp. 921924, 2012. 

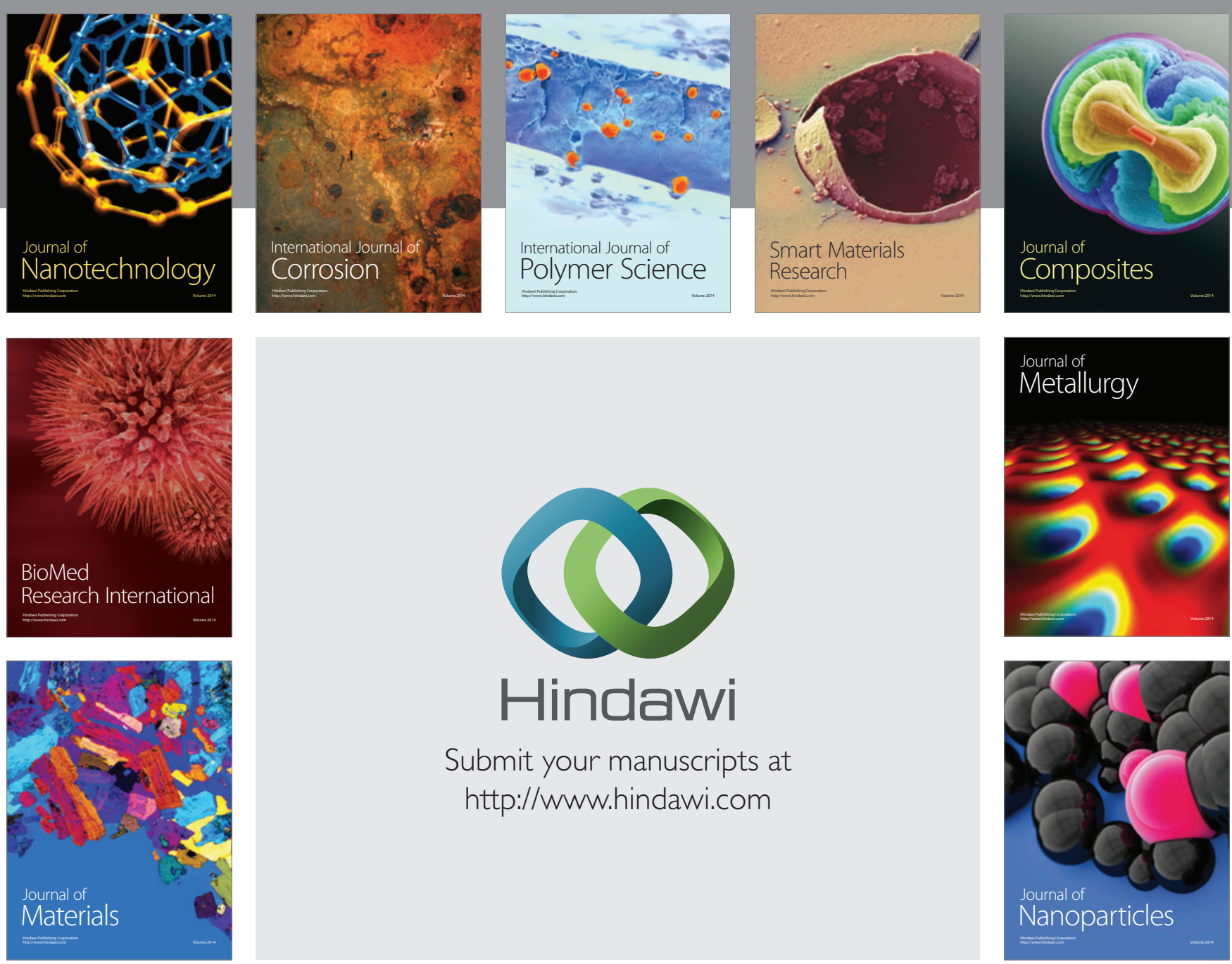

Submit your manuscripts at http://www.hindawi.com
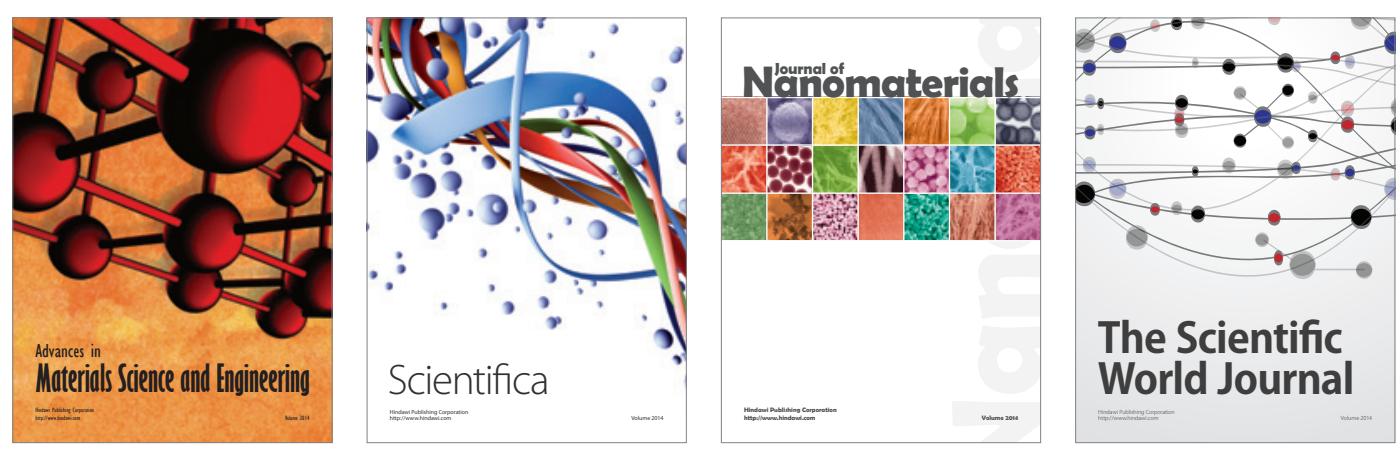

\section{The Scientific World Journal}
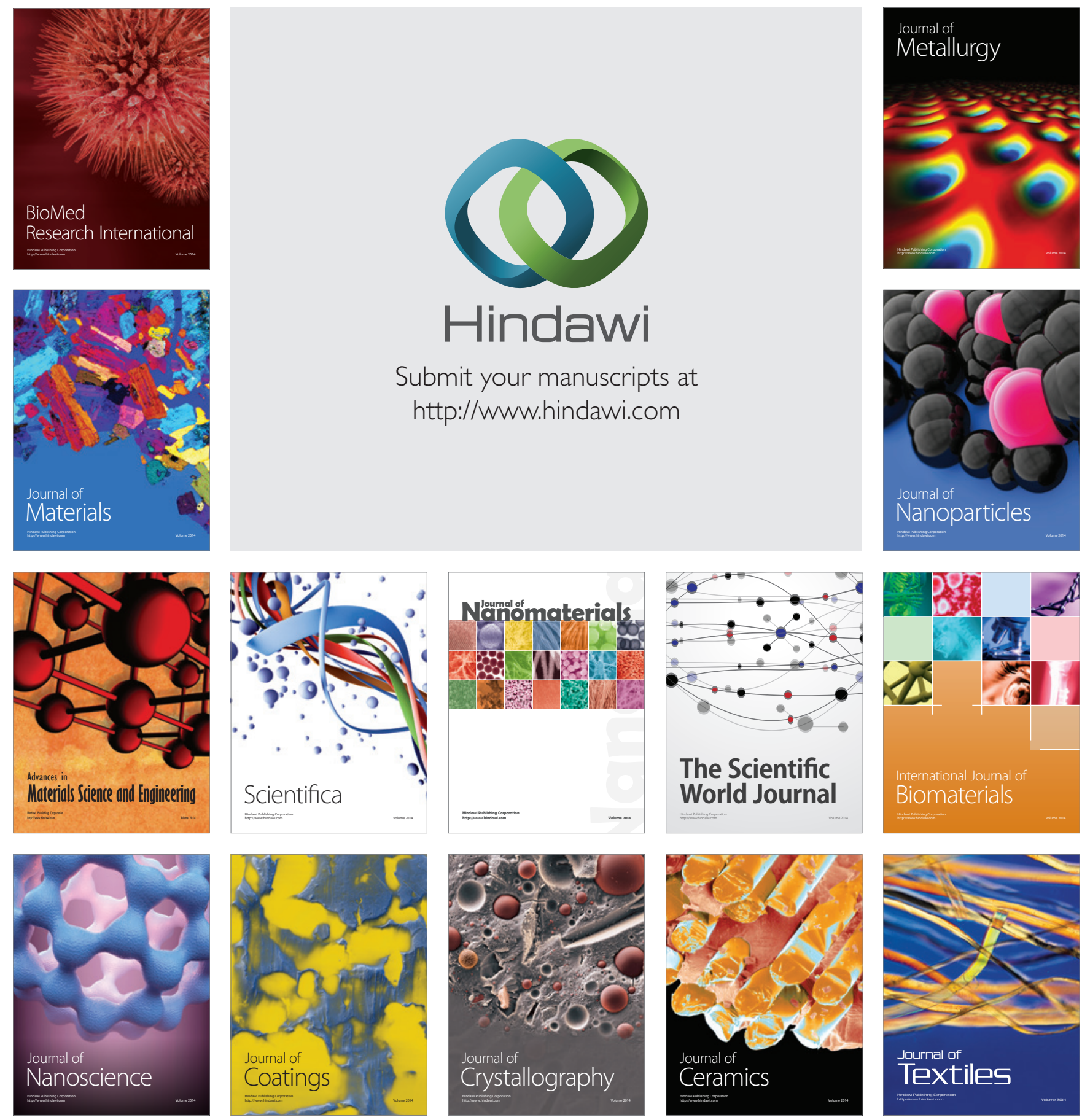